\title{
Topics in Global Public Health
}

\author{
David A. Spiegel MD, Richard A. Gosselin MD, \\ R. Richard Coughlin MD, Adam L. Kushner MD, \\ Stephen B. Bickler MD
}

Published online: 19 August 2008

(C) The Association of Bone and Joint Surgeons 2008

\begin{abstract}
Deficiencies in the delivery of musculoskeletal trauma care in low- and middle-income countries can be attributed to a variety of causes, all of which can be linked to failure of the health system to deliver the necessary services to prevent death and disability. As such, a "systems" approach will be required to improve the delivery of services. The goal of this review is to familiarize the orthopaedic surgeon with selected topics in public health, including health systems, burden of disease, disability adjusted life year (DALY), costeffective analysis, and related concepts (eg, met versus unmet need, access, utilization, effective coverage).
\end{abstract}

\section{Introduction}

While surgeons appropriately focus on the diagnosis and treatment of individual patients, improving the delivery of

\section{A. Spiegel ( $\square)$}

Division of Orthopaedic Surgery, Children's Hospital of Philadelphia, 2nd Floor Wood Building, 34th Street \& Civic Center Blvd, Philadelphia, PA 19104, USA

e-mail: spiegeld@email.chop.edu

\section{R. A. Gosselin, R. R. Coughlin}

University of California at Berkeley School of Public Health, Berkeley, CA, USA

R. A. Gosselin, R. R. Coughlin

University of California at San Francisco, San Francisco, CA, USA

\author{
A. L. Kushner \\ The New York Society of International Humanitarian Surgeons \\ (NYSIHS), New York, NY, USA \\ S. B. Bickler \\ Department of Surgery, University of California at San Diego, \\ San Diego, USA
}

care for surgical diseases requires a population-based approach. Strengthening the delivery of services for musculoskeletal injuries in low- and middle- income countries will require a multidisciplinary, multisectoral approach, which can only be accomplished by addressing particular weaknesses in each health system. Barriers to the delivery of services, which result in an unmet need, include deficiencies in infrastructure, physical resources (supplies and equipment), and human resources for health.

Our goal is to familiarize the orthopaedic surgeon with relevant topics including health systems, burden of disease, disability adjusted life year (DALY), cost-effective analysis, and related concepts (eg, met versus unmet need, access, utilization, effective coverage).

\section{The Health System}

A health system may be defined as "all the activities whose primary purpose is to promote, restore, or maintain health" [34]. Health systems have evolved considerably over the past century. In the early 20th century, the goal in some countries was to provide universal healthcare services. The fundamental premise of universal services was and is that healthcare is a right, not a privilege. However, this laudable early goal was unattainable due to economic constraints. In the midportion of that century, emphasis was placed on the provision of primary healthcare to provide a basic level of services at the population level. The goal of many later 20th century health systems has been to provide universal access to a group of "essential services." Given the financial constraints on health systems, this approach implies that services must be prioritized, and that not all interventions will be financed. How do stakeholders determine which services or interventions are essential? In 
addition to understanding the disease burden (local or national), interventions must be judged on their effectiveness, costs, and social acceptability [13, 34].

Deficiencies in musculoskeletal trauma care may be viewed as a failure of the healthcare system to deliver the necessary services to prevent or reduce death and disability. Using the definition cited above, a health system is charged with prevention, treatment, and rehabilitation following injuries. Injury prevention efforts include improvements in road infrastructure, such as building sidewalks and installing speed bumps, using reflective devices, public education programs, and appropriate legislation to influence driving behavior. Efforts to improve treatment may be aimed at prehospital care, the provision of services once a patient reaches a healthcare facility, and then rehabilitation to maximize function and promote social reintegration.

The World Health Report 2000 emphasized the importance of health systems in promoting good health, responding to the demands of the population, and ensuring that personal financial contributions are commensurate with the ability to pay (prevent impoverishment from personal healthcare expenditures) [34]. A health system has four functions, namely stewardship, financing, resource generation, and service provision [34]. Barriers to the delivery of musculoskeletal trauma care may arise within each component of the system. Adequate musculoskeletal trauma services are often available at private facilities in urban centers, but may be lacking for the majority of the population who have limited financial resources and reside in rural communities.

The stewardship function is most important, and is carried out by governments and their ministries of health. There may also be limited contributions from the private sector and from other sources. In addition to allocating resources for healthcare delivery, the government is responsible for establishing and overseeing health sector policies. A broad range of policies are covered, including the allocation of finances for the health system, regulatory measures (businesses, the pharmaceutical industry, private voluntary organizations and nongovernmental organizations), setting health standards and guidelines, and raising awareness of key health issues [13]. In addition, governmental actions and policies in other areas, including those relating to poverty, education, and the environment, may also have an impact upon health [34]. The development of policies and guidelines pertaining to the prevention and treatment of injuries would seem essential if we are to reduce the burden of injuries and improve care for the injured. The importance of advocacy cannot be understated; global efforts to raise awareness of the burden of injury are essential, and initiatives that aim to strengthen health systems from the standpoint of both prehospital care and treatment in a medical facility should be developed and supported by governments and the global health community. If we are to advocate for system-level changes to improve musculoskeletal trauma care, we must convince the "stewards" that the burden of injuries is large and should be a priority, and that their investment in the prevention and treatment of musculoskeletal injuries will reduce death and disability at a reasonable cost. Epidemiologic data concerning musculoskeletal injuries and their sequelae are lacking in most low- and middle-income countries, despite the fact that injury is now recognized as a global public health concern. Such data will be essential to collect to properly prioritize national goals.

Adequate financing involves both capital investments (one-time startup costs) and recurrent costs (long-term investments to maintain the system) [34]. The basic capacity to deliver services, including infrastructure, physical resources, and human resources, must be available and maintained. Treatment facilities must be adequately equipped and functional. The equipment and supplies needed to care for musculoskeletal injuries (plain radiographs, closed management, irrigation and débridement, skeletal traction, and others) must be maintained. In terms of human resources, there must be an adequate number of trained providers (capital investment). These individuals must be retained (adequate compensation, opportunities for career development) and their knowledge and skills must be maintained and enhanced through continuing education (recurrent cost). There has been a human resource crisis in low- and middle-income countries; in addition to a paucity of trained surgeons, migration of health professionals both within and between countries (brain drain) has been a major problem. Rather than training specialists, many countries have emphasized the provision of multiskilled professionals, especially in rural areas.

Adequate financing must be available to support musculoskeletal trauma care services for each level of health facility, and when governmental sources are insufficient, consideration should be given to public-private partnerships and/or other means of securing funds. Resource generation applies to the basic inputs to the health system, such as physical capital and consumables (for example pharmaceuticals) [34].

Service provision is the main function of each health system, and from a population-based standpoint, the interventions selected must be appropriate to the local pathology, must be affordable and sustainable, and must be socially acceptable. Given a large number of competing health priorities and a limited budget, allocation of resources may be a challenge. Regarding musculoskeletal trauma care, the system must address prehospital care, services during admission to a treatment facility, and rehabilitation of the injured following definitive treatment. 
Systems issues related to prehospital care include the delivery of services at the scene of injury and transport to a treatment facility. In addition to basic life support, responders at the scene should be able to identify major musculoskeletal injuries and prepare patients for transport. A mechanism for communication must be available. There must be adequate road infrastructure and vehicles for transport (ambulance or informal mechanism such as family, taxis, etc.). Once the patient arrives at a treatment facility, depending upon the level of service provided, there must be the capacity to evaluate, stabilize, and ideally treat the injury. When definitive care cannot be provided, there must be a reliable mechanism for referral to higher levels of service. A core group of specific interventions must be developed and supported based upon the level of the health facility and focusing on the nonoperative management of musculoskeletal injuries. While it may be unreasonable to promote locked nailing for fractures of the femur or tibia in resource-constrained environments, the skilled use of skeletal traction should be safe, effective, and affordable. Adequate wound toilet to prevent osteomyelitis following open fractures should be emphasized. A host of other treatments should be well within the capacity of primary health facilities in low- and middle-income countries.

\section{Access to Health Services}

The previous discussion highlights that the delivery of musculoskeletal trauma care is a systems issue and that multiple stakeholders must contribute. Individuals with a musculoskeletal injury should receive safe, timely, and effective treatment. How do we define whether individuals with a musculoskeletal injury in a given population (a district in a low income country, for example) are receiving adequate services from the existing health system (government, private, charity, or nongovernmental organization facilities, or traditional practitioners)? If we define "need" as having a condition which requires treatment to prevent death or disability, then the term "met need" would be used to describe the percentage of patients who received appropriate treatment and "unmet need" would describe those who did not receive appropriate treatment for whatever reason. Other relevant terms include access, utilization, and effectiveness [30]. While there is some variability in how these terms have been defined, the basic concepts are worthy of discussion.

The term "access" implies that patients with a health problem will have the opportunity to be treated. Penchansky and Thomas [28] describe a model of access with four dimensions. A service must be available (volume and type), accessible (patients can physically reach a treatment facility), affordable (size of financial contribution relative to patients ability to pay), and acceptable (relationship between patients attitudes/expectations and the characteristics of those providing services). Even if access is assured within the health system, patients with an injury might not choose to be treated for a variety of reasons. Utilization of health services is not only determined by access, but also by "health behavior"; the patient must recognize the benefits of being treated, must trust that the health system or individual provider is capable of delivering adequate treatment, and must be able to afford the service [30]. Depending upon the circumstances, a patient may elect to be treated by a traditional healer rather than at the district hospital or other facility.

Effective coverage is a recent metric that incorporates all of these concepts and may "clarify the interrelationships between the notions of access, demand for care, utilization, and coverage" [16, 30, 31]. Effective coverage may be defined as the "fraction of potential health gain that could be delivered through an intervention by the health system that is actually delivered" [30]. This tool can be applied to the system as a whole or to specific interventions, and has recently been used to gauge health system performance in Mexico [16]. At the most basic level, effective coverage may be calculated for an individual (I) and an intervention (y), as follows:

$\mathrm{EC}_{\mathrm{iy}}=\mathrm{Q}_{\mathrm{iy}} \times \mathrm{U}_{\mathrm{iy}} / \mathrm{N}_{\mathrm{iy}}$,

where $Q_{i y}$ represents the quality of intervention $y, U_{i y}$ is the probability that the individual will utilize intervention $y$ from an available provider, and $\mathrm{N}_{\mathrm{ij}}$ is the persons with need for that intervention. As mentioned previously, utilization $\left(\mathrm{U}_{\mathrm{iy}}\right)$ depends on a variety of factors, including distance to the treatment center, perception that the problem can be effectively treated, costs, perceived quality of the provider, and cultural acceptability. Ideally, only those with a true need will receive services, but in reality a subset of patients with perceived need, but not true need, will present for treatment.

\section{Burden of Disease and the DALY}

In order to advocate for strengthening the capacity for musculoskeletal services for the injured, it is necessary to assess the impact of musculoskeletal injuries. The costs to the individual, the community, and the society must be quantified relative to other competing health priorities, using a common language. While considerable progress has been made in calling attention to the impact of injuries (fatal and nonfatal) through the Global Burden of Disease Study $[6,15,17,18,26]$, specific data regarding death and disability from musculoskeletal injuries is largely unavailable. 
While mortality has traditionally been the metric of choice in studies of population health, this variable ignores the enormous impact of nonfatal conditions; as such, the impact of most conditions treated by an orthopaedic surgeon was not captured. The seminal Global Burden of Disease study was initiated in 1988, and sponsored by the World Bank, the World Health Organization, and the Harvard School of Public Health [26]. This study acknowledged the importance of nonfatal outcomes and introduced a new metric called the disability adjusted life year (DALY). One goal for the DALY is to quantify the burden of disease with a metric that could be used for costeffective analysis, thereby helping to inform resource allocation within the health sector. Based on the International Classification of Diseases, 135 diseases and injuries were disaggregated into three groups: Group I (communicable diseases, maternal and perinatal conditions, and nutritional deficiencies), Group II (noncommunicable diseases), and Group III (injuries). Injuries are further divided into intentional (eg, self-inflicted violence, interpersonal violence, war) and unintentional (eg, road traffic injuries, poisoning, falls, fires, drowning). Most conditions, while not formally listed, may be categorized as a consequence of one of these diagnoses. For example, obstetric fistula, which causes considerable morbidity in sub-Saharan Africa, is included under the diagnosis of obstructed labor. The results of this ongoing analysis have been published in many forms; the original global burden of disease study was published in 1996 [6, 15, 17, 18, 23, 24, 26]. Individual countries are also pursuing burden of disease analyses. While a voluminous amount of data has been generated, the burden of surgical diseases, including traumatic musculoskeletal conditions, has not been addressed in detail. Injuries accounted for $16 \%$ of the world's disease burden in 1998 and 2002, and $10 \%$ of deaths in low- and middleincome countries (11\% of all DALYs) in 2001 [15, 18]. Unintentional injuries were associated with $70 \%$ of injuryrelated DALYs in 2001, and most occur in males from 15 to 44 years of age [27]. Extremity injuries are recognized as a common source of permanent disability. To our knowledge, however, the data have yet to be disaggregated in existing burden of disease studies [7, 8, 20-22]. The most comprehensive discussion of surgical disease burden is found in the second edition of the Disease Control Priorities in Developing Countries in 2006 [9]. In that analysis based upon data from the World Health Report (2002), conditions that require surgery were estimated to represent $11 \%$ of the world's DALYs. Injuries were believed responsible for $38 \%$ of all surgical DALYs and $4 \%$ of the world's DALYs [9].

The DALY is one summary measure of population health and was developed to capture the combined impact of mortality and morbidity. The DALY reflects the gap between the health of a population and an ideal reference state. One DALY represents a loss of 1 year of healthy life. How is the DALY calculated?

$\mathrm{DALY}=\mathrm{YLL}+\mathrm{YLD}$

The DALY combines time lost due to premature mortality (years of life lost $=$ YLL) and the time lived with disability (years of healthy life lost due to disability = YLD). The years of life lost (YLL) is calculated as the number of deaths multiplied by the standard life expectancy at the age of death (difference between age at death and 82.5 years for females and 80 years for males). These ages are based upon the oldest living population in the world, namely Japan. The years of life lived with a disability is calculated by multiplying the number of cases of disability by the average duration of the disease and by a disability weight (reflects severity of disability),

$\mathrm{YLD}=\mathrm{I} \times \mathrm{DW} \times \mathrm{L}$,

where $\mathrm{I}=$ number of cases, DW = disability weight, and $\mathrm{L}=$ length in years. Disability weights lie within a spectrum from 0 (perfect health) to 1 (death). If we assume that orthopaedic conditions do not directly result in death, then calculations of DALYs will solely involve the YLD component. In addition, the calculation of DALYs also factors in social value choices. Time discounting is a common feature of economic analyses; in the case of DALYs, discounting of $3 \%$ is applied to the years of life lost in the future. A year of healthy life gained is worth $24 \%$ less 10 years from now than 1 year of healthy- life gained at present. Nonuniform age weighting has also been employed in previous burden of disease studies, which gives less weight to the young and the old, emphasizing that society places a maximal value on health of young adults during their prime working age. Disability weights are purported to reflect societal preferences for health states. The values for disability weights were determined by a panel of experts using a Person Trade Off method, and vary between 0 (complete health) and 1 (death) [6, 15, 26, 32]. Examples of disability weights used in the 1990 GBD study include dislocations (0.074), fractures of the femur (0.372), ankle (0.196), and tibia/fibula (0.271)], leg amputations (0.300), osteoarthritis of the hip or knee (0.156), birth asphyxia/ trauma (0.381), tuberculosis (0.294), polio (0.369), spina bifida (0.593), and spinal cord injury (0.725). Disability weights exist for orthopaedic injuries (Table 1) $[6,15,26$, 32] and in most cases these weights are listed for both the untreated and the treated condition. Weights have also been established for different age ranges (0-4, 5-14, 15-44, $45-59,60+)$, however, there are no differences in values between these ages for the musculoskeletal conditions previously listed (Table 1). 
Table 1. Disability weights for orthopaedic injuries

\begin{tabular}{|c|c|c|c|c|}
\hline \multirow[t]{2}{*}{ Type of trauma } & \multicolumn{2}{|c|}{ Short-term weight } & \multicolumn{2}{|c|}{ Long-term weight } \\
\hline & Treated & Untreated & Treated & Untreated \\
\hline \multicolumn{5}{|l|}{ Fractures } \\
\hline Vertebrae & 0.266 & 0.266 & 0 & 0 \\
\hline Pelvis & 0.247 & 0.247 & 0 & 0 \\
\hline $\begin{array}{l}\text { Clavicle, scapula, } \\
\text { or humerus }\end{array}$ & 0.153 & 0.153 & 0 & 0 \\
\hline Femur & 0.372 & 0.372 & 0.272 & 0.272 \\
\hline Radius or ulna & 0.180 & 0.180 & 0 & 0 \\
\hline Hand & 0.100 & 0.100 & 0 & 0 \\
\hline Patella, tibia, or fibula & 0.271 & 0.271 & 0 & 0 \\
\hline Ankle & 0.196 & 0.196 & 0 & 0 \\
\hline Foot & 0.077 & 0.077 & 0 & 0 \\
\hline \multicolumn{5}{|l|}{ Dislocations } \\
\hline Shoulder, elbow, or hip & 0.074 & 0.074 & 0 & 0 \\
\hline Other & 0.074 & 0.074 & 0 & 0 \\
\hline Sprains & 0.064 & 0.064 & 0 & 0 \\
\hline Spinal cord injury & 0 & 0 & 0.725 & 0.725 \\
\hline Injured nerves & 0.064 & 0.078 & 0.064 & 0.078 \\
\hline \multicolumn{5}{|l|}{ Amputations } \\
\hline Thumb & 0.165 & 0.165 & 0.165 & 0.165 \\
\hline Arm & 0.257 & 0.257 & 0.257 & 0.257 \\
\hline Foot & 0.300 & 0.300 & 0.300 & 0.300 \\
\hline Leg & 0.300 & 0.300 & 0.300 & 0.300 \\
\hline
\end{tabular}

Both the methods and assumptions used in calculating DALYs have been criticized on a variety of grounds, and the methodology continues to evolve since the first global burden of disease study was published in 1996 [2-4, 23, 24, 29, 33]. Age weighting has been suggested to devalue the lives of the young and old, and has been discontinued in some recent burden of disease calculations. Another major criticism surrounds the disability weights, which a global social milieu, fail to capture the local contextual conditions in which a given condition may be experienced. For example, a person with a below-knee amputation who lacks access to a prosthesis will likely experience a greater disability than a similar person who has access to a well-fitted prosthesis. One study reported that paraplegic patients in Cameroon rated their health as substantially worse than paraplegics in Australia [1]. Thus burden of disease statistics must be viewed within the context of the geographic location where the disability occurs, including the local infrastructure, and the availability of support from the family, community, and society. Despite these criticisms, the DALY does provide a composite measure allowing comparisons across a variety of conditions and interventions.

\section{Cost-effective Analysis}

Given a large list of competing health priorities, how can health planners determine whether to invest in selected interventions (or a "core group" of services) to treat musculoskeletal injuries? Economic evaluations are timetested tools that are widely used in the health sector. In general, they aim to assess if the benefits from any given intervention outweigh its costs in order to inform and support decision-making in the resource allocation process. Although they should never be the only consideration in the decision-making, their importance cannot be overstated, particularly where resources are scarce. There are three basic types of evaluations: cost-benefit analysis, costeffective analysis, and cost-utility analysis. In a cost-benefit analysis, both costs and benefits are assessed in monetary terms ( $\$ \mathrm{X}$ for a new antibiotic will save $\$ Y$ ).

A cost-effectiveness analysis (CEA) measures the output in natural units, such as lives saved ( $\$ X$ for a new antibiotic will prevent $\mathrm{Y}$ more wound infections) [10]. A cost-utility analysis, where the output reflects a change in the quality of life, is usually measured using one of the many different quality of life (QoL) metrics, such as the DALY, the quality adjusted life year (QALY), or the healthy year equivalent (HYE) to name a few ( $\$ X$ for a new antibiotic will avert $\mathrm{Y}$ DALYs). Most economic evaluations in developing countries have been performed for nonsurgical conditions such as vaccinations, family planning, or nutrition. Only a few evaluations for surgical conditions such as cataracts or obstetrics $[5,11,12,19]$ have been carried out in low-income countries, even though the World Health Organization published clear guidelines on cost-effectiveness analysis in 2000 [25]. Laxminarayan et al. [14] reviewed the findings in the 2nd edition of the Disease Control Priorities in Developing Countries, which suggested that the cost-effectiveness of a basic surgical service was a low-cost opportunity that compared favorably with a many other health initiatives, including primary coverage for maternal and neonatal care, antiretroviral treatment for HIV, and others. The literature is replete with so-called CEAs where results are presented in dollars per DALY averted, or per QALY gained, and are thus truly cost-utility analyses.

How costs are measured will depend on the goal of the analysis. If one wants to compare over time, costs will be measured at the margin: what is the extra cost to produce one more unit of output? One major advantage of economic evaluations is that they allow comparisons across different types of interventions. Since the outputs are different, the costs need to include all inputs, not just the marginal ones. Most evaluations fall in this category and the general principle for calculating costs is as follows: how much would it cost to reproduce from scratch this activity 
somewhere else? Fixed costs such as land, building and equipment need to be amortized and depreciated according to fairly standard schedules. Operating or variable costs include salaries and benefits, consumables, maintenance and administrative costs. The monetary unit (US dollar, International dollar) and time period (eg, monetary value in 2000) should be clearly stated, which is surprisingly not always the case.

The output side of the equation is not as easily measured. Randomized control trials (RCTs) for surgical interventions are almost never conducted in developing countries. Data collection is difficult, and mid- and longterm followup is often unavailable. As such, most studies are retrospective case-control series with their well-known biases. Some surgical conditions (eg, a ruptured spleen) do not require long-term followup if the outcome is measured in terms of death or survival, but most conditions do. Does nailing of tibial fractures produce better functional outcomes than cast treatment? Results from studies in wealthy countries are not necessarily applicable to poor ones. Often in such context, the true outcome of interest cannot be measured and one needs to rely on the next best available data, proxy measures, as the basis for assumptions. The airline analogy can be helpful: outputs of interest could be happy vacations, number of passengers, or number of flights. The first one is very difficult to measure, but is the most important outcome. The second one could serve as a proxy measure, but it is clear that many assumptions must be made. And the third one is irrelevant.

Another problem is when a QoL metric is used, by definition, a certain dose of subjectivity is injected into the equation. The WHO and the World Bank have chosen the DALY as their basic metric, and cost-effectiveness is reported in dollars per DALY averted. It is beyond the scope of this paper to discuss the ethical, moral, or technical considerations underpinning such a choice. As noted previously, DALYs have been criticized on a number of grounds. One major advantage is the ability to compare the cost-effectiveness of interventions that are otherwise difficult, if not impossible, to compare (Table 2). One can compare the cost-effectiveness of tetanus vaccination to

Table 2. Cost-effectiveness of interventions

\begin{tabular}{ll}
\hline Intervention & Costs per DALY averted \\
\hline Tetanus immunization & $\$ 2$ \\
Measles immunization & $\$ 15$ \\
Oral rehydration salts & $\$ 40$ \\
Tibia nailing, 20-year-old male, USA & $\$ 3,800$ \\
THA 60-year-old male, USA & $\$ 48,000$ \\
Cataract surgery, India & $\$ 57$ \\
Surgical trauma, Cambodia & $\$ 78$ \\
\hline
\end{tabular}

measles vaccination, but also to the use of oral rehydration salts (ORS) for diarrheal diseases. Costs, fixed or operating, are highly contextual. A tibial nailing in a 20 -year-old man in the United States costs around $\$ 3,800$ per DALY averted, certainly much higher than it would in Cambodia. But it is still much more cost efficient than a total hip arthroplasty in a 60-year-old man in the United States at around $\$ 48,000$ per DALY averted. Almost all reported CEAs on surgical intervention are from developed countries and for the longest time, public health specialists have thought surgery much too expensive for developing countries. Data has recently emerged to contradict this longstanding dogma: surgery for cataracts in India (\$57/DALY averted) [5], obstetrics in Bangladesh (\$11/DALY averted) [19], a basic surgical service in Sierra Leone (\$38/DALY averted) [12], or a trauma hospital in Cambodia (\$78/ DALY averted) [11] can be extremely cost effective in these particular circumstances. Laxminarayan et al. [14] summarized data from the Disease Control Priorities in Developing Countries and quoted $\$ 70$ to $\$ 210$ per DALY averted for a basic surgical service (injuries, obstetrics, some elective procedures) at the district hospital level. In comparison, dollars per DALY averted for other health interventions were as follows: vaccination $(<\$ 20)$, directly observed therapy (DOTS) for tuberculosis (\$5-35), integrated management of childhood illness (IMCI)(\$38), reproductive health (\$82-148), antiretroviral therapy for AIDS (\$350-500), and pharmacologic treatment for depression (\$478-1288). Further analysis of this nature will be necessary to define a core set of interventions which can be delivered at a reasonable cost in order to reduce the morbidity from musculoskeletal injuries.

\section{Discussion}

Where are we? The epidemic of injuries has resulted in an enormous burden for low and middle oncome countries, and both the mortality and morbidity associated with these injuries undoubtably contributes to the vicious cycle of poverty. Existing health systems have been unable to adequately address this burden, and both the capacity to deliver services and the quality of those services must be enhanced if we are to improve outcomes.

Where are we going? Strengthening the delivery of services for patients with musculoskeletal injuries requires a multidisciplinary approach, and deficiencies in the healthcare system must be addressed to achieve this goal. The burden of injuries must be accurately quantified in a language that permits comparison with competing health priorities. Using a metric such as the DALY, despite it's inherent weaknesses, may facilitate such comparisons. Epidemiologic data must be collected at the local, regional, 
and national levels to gauge the impact of musculoskeletal trauma and identify modifiable risk factors. Cost-effective analysis may help to further define which interventions are most realistic. Methods proven to be safe and effective must be made available at the population level, focusing on areas of unmet need. Existing metrics such as maternal mortality or the under five mortality do not accurately reflect the burden of surgical diseases including musculoskeletal injuries, and are not ideally suited to monitoring interventions designed to improve musculoskeletal trauma care. As such, newer metrics such as effective coverage must be developed.

How do we get there? Orthopaedic surgeons have the opportunity to work with other stakeholders to produce meaningful research, to define essential interventions, and to design and implement strategies to overcome barriers to the delivery of services for the injured in low and middle income countries. A working knowledge of key concepts in public health, and their application in musculoskeletal trauma care, will allow us to work more effectively with others to improve musculoskeletal trauma care.

\section{References}

1. Allotey P, Reidpath DD, Kouame A, Cummins R. The DALY, context and the determinants of the severity of disease: An exploratory comparison of paraplegia in Australia and Cameroon. Soc Sci Med. 2003;57:949-958.

2. Anand S, Hanson K. Disability-adjusted life years: A critical review. J Health Econ. 1997;16:685-702.

3. Arnesen T, Kapirini L. Can the value choices in DALY's influence global priority-setting? Health Policy. 2004;70:137-139.

4. Arnesen T, Nord E. The value of DALY life: Problems with ethics and validity of disability adjusted life years. BMJ. 1999; 319:1423-1425.

5. Baltussen R, Sylla M, Mariotti SP. Cost-effectiveness analysis of cataract surgery: a global and regional analysis. Bull World Health Org. 2004;82:338-345.

6. Begg S, Tomijima N. Global burden of injury in the year 2000: An overview of methods. Available at: http://www.who.int/ entity/healthinfo/statistics/bod_injuries.pdf. Accessed 29 April 2008.

7. Beveridge M, Howard A. The burden of orthopedic disease in developing countries. J Bone Joint Surg Am. 2004;86:1819-1822.

8. Bickler SW, Sanno-Duanda B. Epidemiology of pediatric surgical admissions to a government referral hospital in the Gambia. Bull World Health Org. 2000;78:1330-1336.

9. Debas HT, Gosselin RA, McCord C, Thind A. Surgery. In: Jamison DT, Breman JG, Measham AR, Alleyne G, Claeson M, Evans DB, Jha P, Mills A, Musgrove P, eds. Disease Control Priorities in Developing Countries (2nd ed). New York, NY: Oxford University Press; 2006:1245-1260.

10. Gold MR, Seigel JE, Russel LB, Weinstein MC. Cost-effectiveness in Health and Medicine. New York, NY: Oxford University Press; 1996.

11. Gosselin RA, Heitto M. Cost-effectiveness of a district trauma hospital in Battambang, Cambodia. World J Surg. In press, doi: 10.1007/s00268-008-9708-4.
12. Gosselin RA, Thind A, Bellardinelli A. Cost/DALY averted in a small hospital in Sierra Leone: What is the relative contribution of different services? World J Surg. 2006;30:505-511.

13. Islam $\mathbf{M}$, ed. Health systems assessment approach: A how-to manual. Submitted to the U.S. Agency for International Development in collaboration with Health Systems 20/20, Partners for Health Reformplus, Quality Assurance Program, and Rational Pharmaceutical Management Plus, Arlington, VA: Management Sciences for Health. Available at: http://www.healthsystems2020. org/content/resource/detail/528/). Accessed 8 June 2008.

14. Laxminarayan R, Mills AJ, Breman JG, Measham AR, Alleyne G, Claeson M, Jha P, Musgrove P, Chow J, Shahid-Salles S, Jamison DT. Advancement of global health: key messages from the Disease Control Priorities Project. Lancet. 2006;367: 1193-1208.

15. Lopez AD, Mathers CD, Ezzati M, Jamison DT, Murray CJL, eds. Global Burden of Disease, Risk Factors. New York, NY: World Bank and Oxford University Press; 2006.

16. Lozano R, Soliz P, Gakidou E, Abbott-Klafter J, Feehan DM, Vidal C, Ortiz JP, Murray CJ. Benchmarking of performance of Mexican states with effective coverage. Lancet. 2006;368:1729-1741.

17. Mathers CD, Bernard C, Iburg KM, Inoue M, Ma Fat D, Shibuya $\mathrm{K}$, Stein C, Tomijima N, Xu H. Global Burden of Disease in 2002: data sources, methods, and results. Available at: http:// www.who.int/healthinfo/paper54.pdf. Accessed 29 April 2008.

18. Mathers CD, Loncar D. Projections of global mortality and burden of disease from 2002 to 2030. PLoS Med. 2006;3:e442.

19. McCord C, Chowdhury Q. A cost effective small hospital in Bangladesh: what it can mean for emergency obstetric care. Int $J$ Gynaec Obstet. 2003;81:83-92.

20. Mock CN, Adzotor KE, Conklin E, Denno DM, Jurkovich GJ. Trauma outcomes in the rural developing world: comparison with an urban level I trauma center. J Trauma. 1993;35:518-523.

21. Mock CN, Arreola-Risa C, Quansah R. Strengthening care for injured persons in less developed countries: a case study of Ghana and Mexico. Inj Control Saf Promot. 2003;10:45-51.

22. Mock C, Boland E, Acheampong F, Adjei S. Long-term injury related disability in Ghana. Dis Rehab. 2003;25:723-741.

23. Murray CJL. Quantifying the burden of disease: The technical basis for disability-adjusted life years. Bull World Health Org. 1994;72:429-445.

24. Murray CJL, Acharya AK. Understanding DALYs. J Health Econ. 1997;16:703-730.

25. Murray CJL, Evans DB, Acharya AK, Baltussen R. Development of WHO guidelines on generalized cost-effectiveness analysis. Health Econ. 2000;9:235-251.

26. Murray CJL, Lopez AD, eds. The Global Burden of Disease: A Comprehensive Assessment of Mortality and Disability From Diseases, Injuries, and Risk Factors in 1990 and Projected to 2020. Boston, MA: Harvard School of Public Health; 1996.

27. Norton R, Hyder AA, Bishai D, Peden M. Unintentional injuries. In: Jamison D, Evans D, Alleyne G, Jha P, Breman J, Measham A, et al., eds. Disease Control Priorities in Developing Countries (2nd Ed). New York: Oxford University Press; 2006:737-753.

28. Penchansky R, Thomas JW. The concept of access: Definition and relationship to consumer satisfaction. Medical Care. 1981;19:127-140.

29. Reidpath DD, Allotey P, Kouame A, Cummins R. Measuring health in a vacuum: Examining the disability weight of the DALY. Health Pol Plan. 2003;18:351-356.

30. Shengelia B, Murray CJL, Adams OB. Beyond access and utilization: Defining and measuring health system coverage. In: Murray CJL, Evans DB, eds. Health Syetems Performance Assessment. Geneva, Switzerland: World Health Organization; 2003:221-234 
31. Shengelia B, Tandon A, Adams OB, Muray CJL. Access, utilization, quality, and effective coverage: An integrated conceptual framework and measurement strategy. Soc Sci Med. 2005;61:97-109.

32. WHO annex. Table 3: Disability weights. Available at: http:// www.who.int/entity/healthinfo/bodreferencedisabilityweights.xls. Accessed 29 April 2008.
33. Williams A. Calculating the global burden of disease: Time for a strategic reappraisal? Health Econ. 1999;8:1-8.

34. World Health Report 2000. Health Systems: Improving Performance. Geneva, Switzerland: World Health Organization; 2000. 